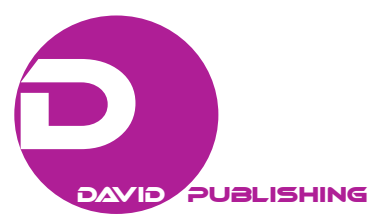

\title{
The Performance Analysis Of A M/M/2/2+1 Retrial Queue With Unreliable Server
}

\author{
R. Kalyanaraman and M. Seenivasan \\ Department of Mathematics, Annamalai University, Annamalainagar-608002, India
}

\begin{abstract}
The $M / M / r / r+d$ retrial queuing system with unreliable server is considered. The customers arrive according to a Poisson process and the service time distribution is negative exponential. The life time of the server and repair times are also negative exponential. If the system is full at the time of arrival of a customer, the customer enters into an orbit. From the orbit the customer tries his luck. The time between two successive retrial follows negative exponential distribution. The model is analyzed using Matrix Geometric Method. The joint distribution of system size and orbit size in steady state is studied. Some system performance measures are obtained. We also provide numerical examples by taking particular values to the parameters.
\end{abstract}

Keywords: Retrial queue, Matrix-Geometric Method, Retrial rate, Stationary distribution, Performance measure.

\section{Introduction}

In a queuing system, if customers arrive for service and find all servers are busy, leave the service area temporarily, stay in orbit and repeat their demand after a random amount of time, such a queuing system is called retrial queuing system. Between trials the customer is called to be in orbit. Retrial queuing theory occupy a prominent role in the performance analysis of wide range of systems in telecommunication network, telephone switching and manufacturing. So, new type of retrial queuing models were proposed and solved by researchers in the field of Mathematics, Statistics, Management and Engineering. For a completed review of main results and the literature of multi server retrial queues, one may refer the monograph by Falin and Templeton (1997). The retrial queues with $r$ servers and $d$ waiting positions are characterized by when $r$ servers are busy, an entering customer occupies a waiting position with total number of waiting positions as $d$. If all the $r+d$ positions are occupiedthe entering customer leaves the system ot enters the orbit of infinite capacity. The $\mathrm{r}$ server retrial queue with $r+d$ system size Markovian is denoted by $M / M / r / r+d$.

Cohen(1957) studied extensively in the $M / M / r / r$ retrial queue. The stationary distribution of multi server retrial queues with particular values on $r$ and $d$ obtained the closed form results. Jonin and Sedol (1970) and Hanschke (1987) derived in explicit formula for the stationary probabilities of the $M / M / 2 / 2$ retrial queue. A bivariate process was introduced by Gomez Corral and Ramalhoto (1999), which represents the joint process of number of servers and the waiting position occupied, and the number of customers in the orbit. They derived a closed form expression for the stationary distribution of the bivariate process using a simple

Corresponding author: M. Seenivasan, Assistant Professor, Annamalai University. Research fields: queuing theory, stochastic process and their applications. E-mail: emseeni@rediffmail.com. 
recursive approach. They also derive the stationary analysis of $\mathrm{M} / \mathrm{M} / 2 / 2+1$ and $\mathrm{M} / \mathrm{M} / 3 / 3$ queues with linear retrial rates as particular cases.

The multi-server retrial queue, based on the physical behavior of the system, which yields an infinitesimal generator with a modified matrix geometric equilibrium probability vector by using a simplified approximation was analyzed by Neuts and Rao (1990). They investigated a multi server retrial queue by using matrix geometric method.

The servers of a retrial queuing models, may subject to failures and repairs. In queuing system, it is normally assumed that the customer whose service is interrupted will be able to complete his service when the interruption is cleared. In the retrial queue, the behavior of the interrupt customer is different. That type of customer might either leaves the system or joins the orbit. Aissani (1988) and Kulkarni and Choi (1990) introduced retrial queues with server failures and repairs. The other notable works related to this queueing model are Aissani and Artalejo (1998), Sherman and Kharoufeh (2006) and Yang and Li (1994). Retrial queuing system with unreliable server was analyzed by Kalyanaraman and Seenivasan (2010).

The content of this paper is the analysis of $M / M / r / r+d, r \in 2$ retrial queue with unreliable server. For this queue the Markov Process $\{X(t): t \geq 0\}$ has been defined and the infinitesimal matrix $\mathrm{Q}$ has been obtained. Using the matrix equation $\mathrm{XQ}=0$ and $\mathrm{Xe}=1$, the solution vector $\mathrm{X}$ is steady state, obtained using the Matrix Geometric Method. The rest of the paper is organized as follows: In section 2, a two server retrial queue, that is $r=2$, has been analyzed. In section 3, a performance measures are calculated in the above model. The model is analyzed using numerical examples are given in section 4 . The last section contains a brief conclusion.

\section{The Model and Analysis}

In this section, we consider two parallel servers are giving service to the customers with one waiting position, the arrival follows Poisson with rate $\lambda$. On arrival if the arriving customer find the system full, he leaves the service areaand enters into an orbit of infinite capacity. From the orbit, the customer tries his luck. The inter-retrial time is negative exponential with rate $\alpha_{i}, i$ being the number of customers in the system, $i \in\{0,1\}$. The service time of each customer is again negative exponential with rate $\varepsilon$. While doing service the server may fail. The servers life time is negative exponential with parameter $\beta$. On failure the server is sent for repair, the repair time is negative exponential with parameter $\delta$. Let $X(t)=(S(t), C(t), Q(t))$ be the state of the process at time $t, C(t)$ is the number of customers both waiting and being served. Therefore, $C(t) \in\{0,1,2,3\}$.

Let $Q(t)$ be the number of customers in the orbit and $Q(t) \in\{0,1,2,3, \ldots\}$.

The elements of the state space of the process

$\{X(t)=(S(t), C(t), Q(t)): t \geq 0\}$ are

$S(t)=0$, the server is idle,

$S(t)=1$, the server is busy with a customer,

$S(t)=2$, two servers busy serving a customer each,

$S(t)=3$, a server fails while serving a customer,

The state space of the process $E$ is $\{\{(0,0, \mathrm{j}): j \geq 0\} \cup\{(1,1, \mathrm{j}),(1,2, \mathrm{j}),(1,3, \mathrm{j}): j \geq 0\} \cup\{(2,2, \mathrm{j}),(2,3, \mathrm{j}):$ $j \geq 0\} \cup\{(3,1, \mathrm{j}),(3,2, \mathrm{j}),(3,3, \mathrm{j}): j \geq 0\}\}$ and retrial rate $\alpha_{i},(\mathrm{i}=0,1,2)$.

In equilibrium, the infinitesimal generator matrix is $Q=\left(q_{a b}\right)$ and is defined as 


$$
Q=\left(\begin{array}{cccccccccc}
B_{0} & A_{2} & 0 & 0 & 0 & 0 & 0 & 0 & 0 & \vdots \\
A_{0} & A_{1} & A_{2} & 0 & 0 & 0 & 0 & 0 & 0 & \vdots \\
0 & A_{0} & A_{1} & A_{2} & 0 & 0 & 0 & 0 & 0 & \vdots \\
\vdots & \vdots & \vdots & \vdots & \vdots & & & & &
\end{array}\right), \text { a tridiagonal matrix }
$$

and the row addresses are defined using

$$
\bar{j}=\{(0,0, j),(1,1, j),(1,2, j),(1,3, j),(2,2, j),(2,3, j),(3,1, j),(3,2, j),(3,3, j)\}: j \geq 0
$$

and again $\bar{j}$ is refined as $\bar{j}=\{(0, j),(1, j),(2, j),(3, j),(4, j),(5, j),(6, j),(7, j),(8, j)\}$

The submatrices of $Q$ are

$$
A_{2}=\left(\begin{array}{lllllllll}
0 & 0 & 0 & 0 & 0 & 0 & 0 & 0 & 0 \\
0 & 0 & 0 & 0 & 0 & 0 & 0 & 0 & 0 \\
0 & 0 & 0 & 0 & 0 & 0 & 0 & 0 & 0 \\
0 & 0 & 0 & \lambda & 0 & 0 & 0 & 0 & 0 \\
0 & 0 & 0 & 0 & 0 & 0 & 0 & 0 & 0 \\
0 & 0 & 0 & 0 & 0 & \lambda & 0 & 0 & 0 \\
0 & 0 & 0 & 0 & 0 & 0 & 0 & 0 & 0 \\
0 & 0 & 0 & 0 & 0 & 0 & 0 & 0 & 0 \\
0 & 0 & 0 & 0 & 0 & 0 & 0 & 0 & \lambda
\end{array}\right)
$$

$A_{1}=\left(\begin{array}{ccccccccc}-\left(\lambda+\alpha_{0}\right) & \lambda & 0 & 0 & 0 & 0 & 0 & 0 & 0 \\ \varepsilon & -\left(\lambda+\alpha_{1}+\beta+\varepsilon\right) & 0 & 0 & \lambda & 0 & \beta & 0 & 0 \\ 0 & 0 & -(\lambda+\delta+\beta) & \lambda & \delta & 0 & 0 & \beta & 0 \\ 0 & 0 & 0 & -(\lambda+\delta+\beta) & 0 & \delta & 0 & 0 & \beta \\ 0 & \varepsilon & 0 & 0 & -\left(\lambda+\varepsilon+\beta+\alpha_{2}\right) & \lambda & 0 & \beta & 0 \\ 0 & 0 & 0 & 0 & \varepsilon & -(\lambda+\varepsilon+\beta) & 0 & 0 & \beta \\ 0 & \delta & 0 & 0 & \lambda & 0 & -\left(\lambda+\delta+\alpha_{1}\right) & 0 & 0 \\ 0 & 0 & 0 & 0 & \delta & 0 & \varepsilon & -(\lambda+\delta+\varepsilon) & \lambda \\ 0 & 0 & 0 & 0 & 0 & \delta & 0 & \varepsilon & -(\lambda+\delta+\varepsilon)\end{array}\right)$

$$
A_{0}=\left(\begin{array}{ccccccccc}
0 & \alpha_{0} & 0 & 0 & 0 & 0 & 0 & 0 & 0 \\
0 & 0 & 0 & 0 & \alpha_{1} & 0 & 0 & 0 & 0 \\
0 & 0 & 0 & 0 & 0 & 0 & 0 & 0 & 0 \\
0 & 0 & 0 & 0 & 0 & 0 & 0 & 0 & 0 \\
0 & 0 & 0 & 0 & 0 & \alpha_{2} & 0 & 0 & 0 \\
0 & 0 & 0 & 0 & 0 & 0 & 0 & 0 & 0 \\
0 & 0 & \alpha_{1} & 0 & 0 & 0 & 0 & 0 & 0 \\
0 & 0 & 0 & 0 & 0 & 0 & 0 & 0 & 0 \\
0 & 0 & 0 & 0 & 0 & 0 & 0 & 0 & 0
\end{array}\right)
$$

and 


$$
B_{0}=\left(\begin{array}{ccccccccc}
-\lambda & \lambda & 0 & 0 & 0 & 0 & 0 & 0 & 0 \\
\varepsilon & -(\lambda+\beta+\varepsilon) & 0 & 0 & \lambda & 0 & \beta & 0 & 0 \\
0 & 0 & -(\lambda+\delta+\beta) & \lambda & \delta & 0 & 0 & \beta & 0 \\
0 & 0 & 0 & -(\lambda+\delta) & 0 & \delta & 0 & 0 & 0 \\
0 & \varepsilon & 0 & 0 & -(\lambda+\beta+\varepsilon) & \lambda & 0 & \beta & 0 \\
0 & 0 & 0 & 0 & \varepsilon & -(\lambda+\beta+\delta) & 0 & 0 & \beta \\
0 & \delta & \lambda & 0 & 0 & 0 & -(\lambda+\delta) & 0 & 0 \\
0 & 0 & 0 & 0 & \delta & 0 & \varepsilon & -(\lambda+\delta+\varepsilon) & \lambda \\
0 & 0 & 0 & 0 & 0 & \delta & 0 & \varepsilon & -(\lambda+\delta+\varepsilon)
\end{array}\right)
$$

The steady state probability vector for $Q$, if it exist, is $X=\left(X_{\overline{0}}, X_{\overline{1}}, X_{\overline{2}}, \ldots\right)$, found by solving

$$
X Q=0 \text { and } X e=1
$$

where $e$ is the column unit vector of appropriate dimensions. The system of linear equations $X Q=0$ is solved using the matrix geometric method (Neuts, 1978). Since the rate matrix $Q$ has a block tridiagonal structure, each $X_{\bar{j}} \quad(j=0,1,2,3, t s)$ is a row vector of order nine, that is, $X_{\bar{j}}=\left(X_{0 j}, X_{1 j}, X_{2 j}, X_{3 j}, X_{4 j}, X_{5 j}, X_{6 j}, X_{7 j}, X_{8 j}\right), j \geq 0$. In the stable case, there exist a matrix R such that

$$
X_{\bar{j}}=X_{\overline{0}} R^{j}, j \geq 1
$$

Now the system $X Q=0$ becomes $X_{\overline{0}} B_{0}+X_{\overline{1}} A_{0}=0$

$$
X_{\overline{0}}\left(B_{0}+R A_{0}\right)=0
$$

and $X_{\bar{j}} A_{2}+X_{j+1} A_{1}+X_{j+2} A_{0}=0$

$$
X_{\overline{0}} R^{j}\left(A_{2}+R A_{1}+R^{2} A_{0}\right)=0, j \geq 1
$$

The vector $X_{\overline{0}}$ is uniquely determined by the equation (4) and the normalizing equation

$$
X_{\overline{0}}(I-R)^{-1} e=1
$$

The matrix $\mathrm{R}$ is the minimal solution to the matrix non-linear equation

$$
A_{2}+R A_{1}+R^{2} A_{0}=0
$$

and it is an irreducible non-negative matrix of spectral radius less than one. The following iterative method can be used to compute $\mathrm{R}$ as follows. 


$$
\begin{gathered}
R_{0}=0 \\
R_{n+1}=-A_{2} A_{1}^{-1}-R_{n}^{2} A_{0} A_{1}^{-1}, n \geq 0
\end{gathered}
$$

For a Markov process with such generators, Neuts (1978) has obtained the stability condition as

$$
\pi A_{2} e<\pi A_{0} e
$$

where the row vector $\pi=\left(\pi_{0}, \pi_{1}, \pi_{2}, \pi_{3}, \pi_{4}, \pi_{5}, \pi_{6}, \pi_{7}, \pi_{8}\right)$ is obtained from the infinitesimal generator $A=A_{0}+A_{1}+A_{2}$

$A=\left(\begin{array}{ccccccccc}-\left(\lambda+\alpha_{0}\right) & \left(\lambda+\alpha_{0}\right) & 0 & 0 & 0 & 0 & 0 & 0 & 0 \\ \varepsilon & -\left(\lambda+\beta+\alpha_{1}+\varepsilon\right) & 0 & 0 & \left(\lambda+\alpha_{1}\right) & 0 & \beta & 0 & 0 \\ 0 & 0 & -(\lambda+\delta+\beta) & \lambda & \delta & 0 & 0 & \beta & 0 \\ 0 & 0 & 0 & -(\delta+\beta) & 0 & \delta & 0 & 0 & \beta \\ 0 & \varepsilon & 0 & 0 & -\left(\lambda+\alpha_{2}+\beta+\varepsilon\right) & \lambda+\alpha_{2} & 0 & \beta & 0 \\ 0 & 0 & 0 & 0 & \varepsilon & -(\beta+\varepsilon) & 0 & 0 & \beta \\ 0 & \delta & \alpha & 0 & \lambda & 0 & -\left(\lambda+\alpha_{1}+\delta\right) & 0 & 0 \\ 0 & 0 & 0 & 0 & \delta & 0 & \varepsilon & -(\lambda+\delta+\varepsilon) & \lambda \\ 0 & 0 & 0 & 0 & 0 & \delta & 0 & \varepsilon & -(\delta+\varepsilon)\end{array}\right)$

It can be shown that $A$ is irreducible and that the row vector $\pi$ is unique such that

$$
\pi A=0 \text { and } \pi e=1
$$

Using equation (11) in equation (12), we have

$$
\begin{gathered}
-\left(\lambda+\alpha_{0}\right) \pi_{0}+\varepsilon \pi_{1}=0 \\
\left(\lambda+\alpha_{0}\right) \pi_{0}-\left(\lambda+\beta+\alpha_{1}\right) \pi_{1}+\varepsilon \pi_{4}+\delta \pi_{6}=0 \\
-(\lambda+\delta+\beta) \pi_{2}+\alpha_{1} \pi_{6}=0 \\
\lambda \pi_{2}-(\delta+\beta) \pi_{3}=0 \\
\left(\lambda+\alpha_{1}\right) \pi_{1}+\delta \pi_{2}-\left(\lambda+\alpha_{2}+\varepsilon+\beta\right) \pi_{4}+\varepsilon \pi_{5}+\lambda \pi_{6}+\delta \pi_{7}=0 \\
\delta \pi_{3}+\left(\lambda+\alpha_{2}\right) \pi_{4}-(\beta+\varepsilon) \pi_{5}+\delta \pi_{8}=0 \\
\beta \pi_{1}-\left(\lambda+\alpha_{1}+\delta\right) \pi_{6}+\varepsilon \pi_{7}=0
\end{gathered}
$$




$$
\begin{gathered}
\beta \pi_{2}+\beta \pi_{4}-(\lambda+\delta) \pi_{7}=0 \\
\beta \pi_{3}+\beta \pi_{5}+\lambda \pi_{7}-(\varepsilon+\delta) \pi_{8}=0 \\
\pi_{0}+\pi_{1}+\pi_{2}+\pi_{3}+\pi_{4}+\pi_{5}+\pi_{6}+\pi_{7}+\pi_{8}=1
\end{gathered}
$$

Solving the above equations, we get

$$
\begin{aligned}
& \pi_{0}=B_{0} \delta_{0} \pi_{2} \\
& \pi_{1}=\delta_{0} \pi_{2} \\
& \pi_{3}=\delta_{1} \pi_{2} \\
& \pi_{4}=\delta_{2} \pi_{2} \\
& \pi_{5}=\delta_{3} \pi_{2} \\
& \pi_{6}=\delta_{4} \pi_{2} \\
& \pi_{7}=\delta_{5} \pi_{2} \\
& \pi_{8}=\delta_{6} \pi_{2} \\
& \pi_{2}=\left(1+\delta_{0}+\delta_{1} \delta_{3}+\delta_{4}+\delta_{5}+\delta_{6}+\delta_{7}+\delta_{8}\right)^{-1}
\end{aligned}
$$

where

$$
\begin{gathered}
B_{0}=\varepsilon /\left(\lambda+\alpha_{0}\right) ; \quad \delta_{0}=-B_{6} ; \delta_{1}=-\lambda / a_{3} ; \delta_{2}=-a_{10} B_{4} ; \\
\delta_{3}=-\left(\beta a_{11}+\lambda B_{7}+B_{8} \varepsilon\right) / \beta ; \quad \delta_{4}=a_{10} ; \delta_{5}=B_{7} ; \\
\delta_{6}=-B_{8} ; \quad a_{0}=-\left(\lambda+\beta+\alpha_{1}\right) ; \quad a_{1}=-(\lambda+\delta+\beta) ; \quad a_{2}=\alpha_{1} ; a_{3}=-(\beta+\delta) ; \\
a_{4}=-\left(\lambda+\alpha_{2}+\beta+\varepsilon\right) ; \quad a_{5}=\left(\lambda+\alpha_{2}\right) ; \quad a_{6}=-(\beta+\varepsilon) ; \quad a_{7}=-\left(\lambda+\alpha_{1}+\delta\right) ; \\
a_{8}=-(\lambda+\delta) ; \quad a_{9}=-(\varepsilon+\delta) ; \quad a_{10}=-a_{1} / a_{2} ; \quad a_{11}=-\lambda / a_{3} ; a_{18}=\beta \varepsilon ;
\end{gathered}
$$




$$
\begin{gathered}
a_{19}=\beta \delta-a_{0} a_{7} ; \quad a_{20}=-a_{0} \varepsilon ; \quad B_{1}=a_{18} \varepsilon ; \quad B_{2}=\left(a_{19} \varepsilon+a_{7} a_{20}\right) ; \quad B_{3}=-\beta a_{20} ; \\
B_{4}=\left(B_{3} \delta-B_{2} a_{0}\right) /\left(B_{3} \varepsilon-B_{1} a_{0}\right) ; \quad B_{5}=\left(a_{11} \delta+B_{4} a_{5} a_{10}\right) ; \\
B_{6}=-\left(a_{10} \varepsilon B_{4}+a_{10} \delta\right) / a_{0} ; \quad B_{7}=-\left(\beta B_{6}+a_{10} a_{7}\right) / \varepsilon ; \\
B_{8}=\left(a_{11} \beta+B_{6} \beta+B_{4}\right) / a_{9}
\end{gathered}
$$

and the stability condition takes the form

$$
\lambda \pi_{0}+\alpha_{1}\left(\pi_{1}+\pi_{6}\right)<\lambda\left(\pi_{3}+\pi_{5}+\pi_{8}\right)
$$

Equation (23) to (31) gives the steady state probabilities of $A$.

\section{Performance Measures}

Using the Probability Vectors $X_{\bar{j}}(j \in\{0,1,2,3, \ldots\})$ the following performance measures can be calculated for the model $M / M / 2 / 2+1$.

The performance measures are

(i) The idle Probability $=\sum_{j=0}^{\infty} X_{0 j}$

(ii) $\operatorname{Pr}\{$ One Server is busy $\}=\sum_{j=0}^{\infty}\left\{X_{1 j}+X_{2 j}+X_{3 j}+X_{6 j}+X_{7 j}+X_{8 j}\right\}$;

(iii) $\operatorname{Pr}\{$ Two Servers are busy $\}=\sum_{j=0}^{\infty}\left\{X_{4 j}+X_{5 j}\right\}$;

(iv) $\operatorname{Pr}\{$ A Server is in repair condition $\}=\sum_{j=0}^{\infty}\left\{X_{2 j}+X_{3 j}+X_{6 j}+X_{7 j}+X_{8 j}\right\}$;

(v) Blocking Probability $=\sum_{j=0}^{\infty}\left\{X_{3 j}+X_{5 j}+X_{8 j}\right\}$.

(vi) The Mean number of customers in the orbit $=L=\sum_{i=0}^{\infty} i X_{i} e$

(vii) The Variance number of customers in the orbit $=\mathrm{V}=\sum_{i=0}^{\infty} i^{2} X_{i} e-(L)^{2}$

(viii) Mean Number of Customers in the orbit

when the server is idle $N_{0}=\sum_{j=0}^{\infty} j X_{0 j}$

when one server is busy $N_{1}=\sum_{j=0}^{\infty} j\left\{X_{1 j}+X_{2 j}+X_{3 j}+X_{6 j}+X_{7 j}+X_{8 j}\right\}$;

when two servers are busy $N_{2}=\sum_{j=0}^{\infty} j\left\{X_{4 j}+X_{5 j}\right\}$ 
when the server is in repair $N_{3}=\sum_{j=0}^{\infty} j\left\{X_{2 j}+X_{3 j}+X_{6 j}+X_{7 j}+X_{8 j}\right\}$

\section{Numberical Study}

Some numerical results related to the model discussed in the above section 2 is presented here. By varying $\lambda$ 's, $\alpha_{i}$ 's,$\delta$ 's, $\beta$ 's and $\varepsilon$ 's, three different examples called Example 1, Example 2 and Example 3 ofthe model, are given below.

Example 1: $\quad$ For $\lambda=0.75, \alpha_{0}=0.1, \alpha_{1}=0.9, \alpha_{2}=0.6, \alpha_{3}=0.1, \varepsilon=0.5, \beta=0.01$, $\delta=0.01, \alpha=1$ and the $R$ matrix is given by

$$
R=\left(\begin{array}{lllllllll}
0.0000 & 0.0000 & 0.0000 & 0.0000 & 0.0000 & 0.0000 & 0.0000 & 0.0000 & 0.0000 \\
0.0000 & 0.0000 & 0.0000 & 0.0000 & 0.0000 & 0.0000 & 0.0000 & 0.0000 & 0.0000 \\
0.0000 & 0.0000 & 0.0000 & 0.0000 & 0.0000 & 0.0000 & 0.0000 & 0.0000 & 0.0000 \\
0.0000 & 0.0280 & 0.0063 & 0.9802 & 0.0930 & 0.2129 & 0.0020 & 0.0060 & 0.0130 \\
0.0000 & 0.0000 & 0.0000 & 0.0000 & 0.0000 & 0.0000 & 0.0000 & 0.0000 & 0.0000 \\
0.0000 & 0.1070 & 0.0077 & 0.0075 & 0.3551 & 0.9723 & 0.0030 & 0.0078 & 0.0124 \\
0.0000 & 0.0000 & 0.0000 & 0.0000 & 0.0000 & 0.0000 & 0.0000 & 0.0000 & 0.0000 \\
0.0000 & 0.0000 & 0.0000 & 0.0000 & 0.0000 & 0.0000 & 0.0000 & 0.0000 & 0.0000 \\
0.0000 & 0.0130 & 0.1787 & 0.1741 & 0.0413 & 0.0816 & 0.0942 & 0.3126 & 0.7833
\end{array}\right)
$$

Using this $R$ matrix, $X_{\overline{0}}$ is calculated from the relation $X_{\overline{0}}\left(B_{0}+R A_{0}\right)=0$ and the normalization condition $X_{\overline{0}}(I-R)^{-1} e=1$. The remaining vectors $X_{\bar{i}}, \mathrm{i}=1,2,3 \ldots$. are obtained from $X_{\bar{i}}=X_{\overline{0}} R^{i}$, $i=1,2,3, \ldots$ and are presented in the tables 5.1 and 5.2. In the table rows 2,3,4,5 6,7,8,9,10 and 11 represents the nine components of $X_{\bar{i}}, i=0,1,2, \ldots$ the last row represents the sum of the eight components. It is verified that the total probability is $0.998486 \approx 1$.

Table 5.1

Probability Vectors

\begin{tabular}{|c|c|c|c|c|c|c|}
\hline & $X_{\overline{0}}$ & $X_{\overline{1}}$ & $X_{\overline{2}}$ & $X_{\overline{3}}$ & $X_{\overline{4}}$ & $X_{\overline{5}}$ \\
\hline & 0.000031 & 0.000131 & 0.000129 & 0.000125 & 0.000111 & 0.000102 \\
\hline & 0.000426 & 0.012370 & 0.002262 & 0.001341 & 0.000465 & 0.000395 \\
\hline & 0.002592 & 0.000168 & 0.000133 & 0.000107 & 0.000087 & 0.000174 \\
\hline & 0.000142 & 0.001669 & 0.000293 & 0.000139 & 0.000046 & 0.000023 \\
\hline & 0.000089 & 0.000039 & 0.000027 & 0.000011 & 0.000007 & 0.000003 \\
\hline & 0.000276 & 0.000777 & 0.000171 & 0.000076 & 0.000038 & 0.000017 \\
\hline & 0.000481 & 0.000089 & 0.000070 & 0.000056 & 0.000045 & 0.000037 \\
\hline & 0.024781 & 0.000295 & 0.000233 & 0.000185 & 0.000149 & 0.000021 \\
\hline & 0.947865 & 0.000074 & 0.000058 & 0.000046 & 0.000037 & 0.000014 \\
\hline Total & 0.975652 & 0.015481 & 0.003247 & 0.001961 & 0.000874 & 0.000684 \\
\hline
\end{tabular}

Example 2: $\quad$ For $\lambda=0.85, \alpha_{0}=0.1, \alpha_{1}=0.9, \alpha_{2}=0.6, \alpha_{3}=0.1, \varepsilon=0.5, \beta=0.01$, $\delta=0.01, \alpha=1$ and the $R$ matrix is given by 


$$
R=\left(\begin{array}{lllllllll}
0.0000 & 0.0000 & 0.0000 & 0.0000 & 0.0000 & 0.0000 & 0.0000 & 0.0000 & 0.0000 \\
0.0000 & 0.0000 & 0.0000 & 0.0000 & 0.0000 & 0.0000 & 0.0000 & 0.0000 & 0.0000 \\
0.0000 & 0.0000 & 0.0000 & 0.0000 & 0.0000 & 0.0000 & 0.0000 & 0.0000 & 0.0000 \\
0.0000 & 0.0213 & 0.0046 & 0.8214 & 0.0653 & 0.1482 & 0.0016 & 0.0026 & 0.0546 \\
0.0000 & 0.0000 & 0.0000 & 0.0000 & 0.0000 & 0.0000 & 0.0000 & 0.0000 & 0.0000 \\
0.0000 & 0.2154 & 0.0029 & 0.0046 & 0.2458 & 0.4785 & 0.0082 & 0.0046 & 0.0089 \\
0.0000 & 0.0000 & 0.0000 & 0.0000 & 0.0000 & 0.0000 & 0.0000 & 0.0000 & 0.0000 \\
0.0000 & 0.0000 & 0.0000 & 0.0000 & 0.0000 & 0.0000 & 0.0000 & 0.0000 & 0.0000 \\
0.0000 & 0.0254 & 0.2658 & 0.2458 & 0.0354 & 0.0746 & 0.0248 & 0.3254 & 0.6542
\end{array}\right)
$$

Using this $R$ matrix, $X_{\overline{0}}$ is calculated from the relation $X_{\overline{0}}\left(B_{0}+R A_{0}\right)=0$ and the normalization condition $X_{\overline{0}}(I-R)^{-1} e=1$. The remaining vectors $X_{\bar{i}}, \mathrm{i}=1,2,3 \ldots$ are obtained from $X_{\bar{i}}=X_{\overline{0}} R^{i}$, $i=1,2,3, \ldots$ and are presented in the tables 5.3 and 5.4. In the table rows 2,3,4,5 6,7,8,9,10 and 11 represents the nine components of $X_{\bar{i}}, i=0,1,2, \ldots$ the last row represents the sum of the eight components. It is verified that the total probability is $0.992175 \approx 1$.

Table 5.2

Probability Vectors

\begin{tabular}{lllllll}
\hline & $X_{\overline{6}}$ & $X_{\overline{7}}$ & $X_{\overline{8}}$ & $X_{\overline{9}}$ & $X_{10}$ & $X_{11}$ \\
\hline & 0.000044 & 0.000022 & 0.000008 & 0.000004 & 0.000002 & 0.000001 \\
& 0.000084 & 0.000044 & 0.000031 & 0.000018 & 0.000008 & 0.000005 \\
& 0.000056 & 0.000039 & 0.000010 & 0.000009 & 0.000006 & 0.000002 \\
& 0.000015 & 0.000006 & 0.000004 & 0.000003 & 0.000001 & 0.000000 \\
& 0.000001 & 0.000000 & 0.000000 & 0.000000 & 0.000000 & 0.000000 \\
& 0.000013 & 0.000007 & 0.000005 & 0.000002 & 0.000001 & 0.000000 \\
& 0.000035 & 0.000033 & 0.000014 & 0.000008 & 0.000003 & 0.000001 \\
& 0.000011 & 0.000008 & 0.000005 & 0.000003 & 0.000001 & 0.000000 \\
Total & 0.000009 & 0.000005 & 0.000003 & 0.000001 & 0.000000 & 0.000000 \\
\hline
\end{tabular}

Table 5.3

Probability Vectors

\begin{tabular}{|c|c|c|c|c|c|c|}
\hline & $X_{\overline{0}}$ & $X_{\overline{1}}$ & $X_{\overline{2}}$ & $X_{\overline{3}}$ & $X_{\overline{4}}$ & $X_{\overline{5}}$ \\
\hline & 0.000021 & 0.000068 & 0.000060 & 0.000055 & 0.000048 & 0.000034 \\
\hline & 0.000254 & 0.004663 & 0.003191 & 0.001070 & 0.000310 & 0.000290 \\
\hline & 0.004521 & 0.000147 & 0.000121 & 0.000092 & 0.000068 & 0.000059 \\
\hline & 0.000287 & 0.002416 & 0.000252 & 0.000112 & 0.000038 & 0.000019 \\
\hline & 0.000124 & 0.000062 & 0.000032 & 0.000015 & 0.000008 & 0.000002 \\
\hline & 0.000354 & 0.000652 & 0.000152 & 0.000062 & 0.000034 & 0.000014 \\
\hline & 0.000216 & 0.000081 & 0.000062 & 0.000052 & 0.000039 & 0.000031 \\
\hline & 0.015894 & 0.000146 & 0.000133 & 0.00085 & 0.000049 & 0.000019 \\
\hline & 0.944681 & 0.000081 & 0.000062 & 0.000052 & 0.000028 & 0.000012 \\
\hline Total & 0.966331 & 0.008337 & 0.004065 & 0.001595 & 0.000874 & 0.000480 \\
\hline
\end{tabular}


Table 5.4

Probability Vectors

\begin{tabular}{|c|c|c|c|c|c|c|}
\hline & $X_{\overline{6}}$ & $X_{\overline{7}}$ & $X_{\overline{8}}$ & $X_{\overline{9}}$ & $X_{10}$ & $X_{11}$ \\
\hline & 0.000022 & 0.000018 & 0.000008 & 0.0 & 0.0 & 0.0 \\
\hline & 0.000086 & 0.000050 & 0.000021 & 0.000017 & 0.000008 & 0.000006 \\
\hline & 0.000051 & 0.000022 & 0.000016 & 0.000008 & 0.000005 & 0.000003 \\
\hline & 0.000012 & 0.000004 & 0.000003 & 0.000002 & 0.000000 & 0.000000 \\
\hline & 0.000001 & 0.000 & 0.000000 & 0.00000 & 0.000000 & 0.000000 \\
\hline & 0.000012 & 0.000006 & 0.000004 & 0.000001 & 0.000000 & 0.000000 \\
\hline & 0.000027 & 0.000021 & 0.000012 & 0.000007 & 0.000002 & 0.000001 \\
\hline & 0.000010 & 0.000007 & 0.000004 & 0.000002 & 0.000001 & 0.000000 \\
\hline & 0.000007 & 0.000003 & 0.000002 & 0.000001 & 0.000000 & 0.000000 \\
\hline Total & 0.000228 & 0.000131 & 0.000070 & 0.000038 & 0.000016 & 0.000010 \\
\hline
\end{tabular}

Example 3: $\quad$ For $\lambda=0.95, \alpha_{0}=0.1, \alpha_{1}=0.9, \alpha_{2}=0.6, \alpha_{3}=0.1, \varepsilon=0.5, \beta=0.01$, $\delta=0.01, \alpha=1$ and the $R$ matrix is given by

$$
R=\left(\begin{array}{lllllllll}
0.0000 & 0.0000 & 0.0000 & 0.0000 & 0.0000 & 0.0000 & 0.0000 & 0.0000 & 0.0000 \\
0.0000 & 0.0000 & 0.0000 & 0.0000 & 0.0000 & 0.0000 & 0.0000 & 0.0000 & 0.0000 \\
0.0000 & 0.0000 & 0.0000 & 0.0000 & 0.0000 & 0.0000 & 0.0000 & 0.0000 & 0.0000 \\
0.0000 & 0.0019 & 0.0011 & 0.9783 & 0.0066 & 0.0173 & 0.0009 & 0.0032 & 0.0093 \\
0.0000 & 0.0000 & 0.0000 & 0.0000 & 0.0000 & 0.0000 & 0.0000 & 0.0000 & 0.0000 \\
0.0000 & 0.0919 & 0.0019 & 0.0021 & 0.3235 & 0.9936 & 0.0017 & 0.0061 & 0.0111 \\
0.0000 & 0.0000 & 0.0000 & 0.0000 & 0.0000 & 0.0000 & 0.0000 & 0.0000 & 0.0000 \\
0.0000 & 0.0000 & 0.0000 & 0.0000 & 0.0000 & 0.0000 & 0.0000 & 0.0000 & 0.0000 \\
0.0000 & 0.0034 & 0.0826 & 0.0897 & 0.0105 & 0.0208 & 0.0778 & 0.2738 & 0.7977
\end{array}\right)
$$

Using this $R$ matrix, $X_{\overline{0}}$ is calculated from the relation $X_{\overline{0}}\left(B_{0}+R A_{0}\right)=0$ and the normalization condition $X_{\overline{0}}(I-R)^{-1} e=1$. The remaining vectors $X_{\bar{i}}, \mathrm{i}=1,2,3 \ldots$. are obtained from $X_{\bar{i}}=X_{\overline{0}} R^{i}$, $i=1,2,3, \ldots$ and are presented in the tables 5.5, and 5.6. In the table rows 2,3,4,5 6,7,8,9,10 and 11 represent the nine components of $X_{\bar{i}}, i=0,1,2, \ldots$ the last row represents the sum of the eight components. It is verified that the totalprobability is $0.997254 \approx 1$.

The performance measures for the numerical models related to examples 1,2,3 are calculated, for the model $M / M / 2 / 2+1$ the idle probabilities, the blocking probabilities, probability that one server are busy, the total retrial rate, and the mean number of customers in the orbit are given in the table 5.7.

The mean number of customers in orbit is also too high in the case of two server queues. The results show that the number of servers make substantial changes in the performance measures. 
Table 5.5

Probability Vectors

\begin{tabular}{|c|c|c|c|c|c|c|}
\hline & $X_{\overline{0}}$ & $X_{\overline{1}}$ & $X_{\overline{2}}$ & $X_{\overline{3}}$ & $X_{\overline{4}}$ & $X_{\overline{5}}$ \\
\hline & 0.000080 & 0.000064 & 0.000044 & 0.000027 & 0.000020 & 0.000012 \\
\hline & 0.000088 & 0.000180 & 0.000110 & 0.000022 & 0.000021 & 0.000017 \\
\hline & 0.000248 & 0.001743 & 0.001502 & 0.001307 & 0.000850 & 0.000124 \\
\hline & 0.003824 & 0.0013891 & 0.012076 & 0.011045 & 0.006841 & 0.001498 \\
\hline & 0.000148 & 0.000890 & 0.000639 & 0.000405 & 0.000184 & 0.000097 \\
\hline & 0.000168 & 0.002291 & 0.001640 & 0.000042 & 0.000024 & 0.000012 \\
\hline & 0.000388 & 0.000981 & 0.000842 & 0.000729 & 0.000638 & 0.000565 \\
\hline & 0.009437 & 0.003647 & 0.003126 & 0.002702 & 0.002359 & 0.002083 \\
\hline & 0.846258 & 0.010598 & 0.009065 & 0.007814 & 0.006799 & 0.005981 \\
\hline Total & 0.860639 & 0.034285 & 0.029044 & 0.024996 & 0.018336 & 0.010489 \\
\hline
\end{tabular}

Table 5.6

Probability Vectors

\begin{tabular}{|c|c|c|c|c|c|c|}
\hline & $X_{\overline{6}}$ & $X_{\overline{7}}$ & $X_{\overline{8}}$ & $X_{\overline{9}}$ & $X_{10}$ & $X_{11}$ \\
\hline & 0.000008 & 0.000002 & 0.000001 & 0.0 & 0.0 & 0.0 \\
\hline & 0.000003 & 0.000002 & 0.000001 & 0.000001 & 0.000000 & 0.000000 \\
\hline & 0.000086 & 0.000049 & 0.000021 & 0.000008 & 0.000004 & 0.000001 \\
\hline & 0.000424 & 0.000355 & 0.000089 & 0.000023 & 0.000009 & 0.000004 \\
\hline & 0.000061 & 0.000032 & 0.000014 & 0.000008 & 0.000004 & 0.000000 \\
\hline & 0.000009 & 0.000007 & 0.000003 & 0.000002 & 0.000001 & 0.000001 \\
\hline & 0.000507 & 0.000462 & 0.000427 & 0.000201 & 0.000082 & 0.000030 \\
\hline & 0.001864 & 0.001691 & 0.000558 & 0.000257 & 0.000042 & 0.000012 \\
\hline & 0.005326 & 0.004808 & 0.001403 & 0.000294 & 0.000083 & 0.000029 \\
\hline Total & 0.008368 & 0.007428 & 0.002522 & 0.000794 & 0.000225 & 0.000128 \\
\hline
\end{tabular}

Table 5.7

Performance Measures

\begin{tabular}{lllll}
\hline No. & Measures for Model & Example1 & Example2 & Example3 \\
\hline 1. & The idle Probability & 0.000925 & 0.000457 & 0.000352 \\
2. & Pr $\{$ One server is busy\} & 0.032546 & 0.195482 & 0.042657 \\
3. & Pr \{Two servers are busy\} & 0.084567 & 0.452581 & 0.098745 \\
4. & Blocking Probability & 0.065842 & 0.384525 & 0.024587 \\
5. & Pr \{A server is in repair condition\} & 0.068254 & 0.085721 & 0.465821 \\
6. & The Mean number of Customers in the orbit $(\mathrm{L})$ & 0.18457 & 0.14702 & 0.27935 \\
7. & The Variance number of Customers in the orbit & 0.25648 & 0.34854 & 0.32548 \\
8. & The Mean number of Customers in the orbit & & & \\
& when the server is idle $\left(N_{0}\right)$ & 0.92587 & 0.96542 & 0.80874 \\
& when one server is busy $\left(N_{1}\right)$ & 0.82458 & 0.83532 & 0.81501 \\
& when two servers are busy $\left(N_{2}\right)$ & 0.85842 & 0.72142 & 0.82457 \\
& when three servers are busy $\left(N_{3}\right)$ & 0.87548 & 0.97214 & 0.92501 \\
\hline
\end{tabular}




\section{Conclusion}

In this paper we have considered a multi server retrial queuing system. We have obtained the steady state probability vector by applying matrix geometric method. Using the steady state probability vector, the mean number of customers in the orbithas been obtained. Furthermore, we have performed numerical analysis by assuming particular values to the parameter.

\section{References}

Aissani.A On the M/G/1/1 queueing system with repeated orders and unreliable server, Journal of Technology 6, 98-123, 1988

Aissani.A and J.R. Artalejo, On the sigle server retrial queue subject to breakdowns, Queueing Systems 30, 307-321, 1998

Cohen, J.W. Basic problems of telephone traffic theory and the influence of repeated calls, phillips Telecommunication Review 18, 49-100, 1957.

Falin, G.I. and J.G.C. Templeton, Retrial Queues, Chapman and Hall, 1997.

Gomez-Corral, A. and M.F.Ramalhoto, The stationary distribution of a Markovian process arising in the theory of multi server retrial queuing systems, Mathematical and Computer Modeling 30, 14-158,1999.

Hanschke, T. Explicit formulas for the characteristics of the $M / M / 2 / 2$ queue with repeated attempts, Journal of Applied Probability 24, 486-494, 1987.

Jonin, G.L. and J.J. Sedol, Telephone systems with repeated calls, In proceedings of the 6-th International Teletraffic Congress, Munich, 435(1-5), 1970.

Kalyanaraman, R. and M.Seenivasan, Multi-server retrial queuing system with unreliable server, International Journal of computational cognition Vol 7, No 3, 113-120, 2010.

Kulkarni, V.G. and B.D. Choi, Retrial queues with server subject to breakdowns, Queueing Systems 7, 191-208, 1990.

Neuts, M.F., Markov chains with applications in queuing theory,which have Matrix- Geometric invariant probability vector,Adv.Appl.Probab. 10, 185-212, 1978.

Neuts, M.F. and B.M. Rao, Numerical investigation of a multi server retrial model, Queuing Systems 7, 167-189, 1990.

Sherman, N.P. and J.P. Kharoufeh, An M/M/1 retrial queue with unreliable server, Operations Research Letters 34,697-705, 2006. Yang, T. and H.Li., The M/G/1 retrial queue with the server subject to starting failures, Queueing Systems 16, 83-96, 1994. 